\title{
API5 induces cisplatin resistance through FGFR signaling in human cancer cells
}

\author{
Han Sol Jang ${ }^{1,2,3,7}$, Seon Rang Woo ${ }^{1,2,4,7}$, Kwon-Ho Song ${ }^{1,2,3,7}$, Hanbyoul Cho ${ }^{5}$, Doo Byung Chay ${ }^{5}$, \\ Soon-Oh Hong ${ }^{1,2,3}$, Hyo-Jung Lee ${ }^{1,2,3}$, Se Jin $\mathrm{Oh}^{1,2,3}$, Joon-Yong Chung ${ }^{6}$, Jae-Hoon $\mathrm{Kim}^{5}$ and \\ Tae Woo Kim ${ }^{1,2,3,4}$
}

\begin{abstract}
Most tumors frequently undergo initial treatment with a chemotherapeutic agent but ultimately develop resistance, which limits the success of chemotherapies. As cisplatin exerts a high therapeutic effect in a variety of cancer types, it is often used in diverse strategies, such as neoadjuvant, adjuvant and combination chemotherapies. However, cisplatin resistance has often manifested regardless of cancer type, and it represents an unmet clinical need. Since we found that API5 expression was positively correlated with chemotherapy resistance in several specimens from patients with cervical cancer, we decided to investigate whether API5 is involved in the development of resistance after chemotherapy and to explore whether targeting API5 or its downstream effectors can reverse chemo-resistance. For this purpose, cisplatin-resistant cells (CaSki P3 CR) were established using three rounds of in vivo selection with cisplatin in a xenografted mouse. In the CaSki P3 CR cells, we observed that API5 acted as a chemo-resistant factor by rendering cancer cells resistant to cisplatin-induced apoptosis. Mechanistic investigations revealed that API5 mediated chemoresistance by activating FGFR1 signaling, which led to Bim degradation. Importantly, FGFR1 inhibition using either an siRNA or a specific inhibitor disrupted cisplatin resistance in various types of API $5^{\text {high }}$ cancer cells in an in vitro cell culture system as well as in an in vivo xenograft model. Thus, our results demonstrated that API5 promotes chemo-resistance and that targeting either API5 or its downstream FGFR1 effectors can sensitize chemo-refractory cancers.
\end{abstract}

Experimental \& Molecular Medicine (2017) 49, e374; doi:10.1038/emm.2017.130; published online 8 September 2017

\section{INTRODUCTION}

In general, traditional therapies such as surgery, chemotherapy and radiotherapy are implemented to treat many cancer types. Among these, chemotherapy is frequently used in diverse strategies, including neoadjuvant, adjuvant and combination chemotherapies. ${ }^{1}$ Many patients with different types of cancers (for example, cervical, head and neck, non-small cell lung, gastric and bladder cancer) have been preferentially treated with cisplatin chemotherapy. ${ }^{2-4}$ Cisplatin (cis-diaminedichloroplatinum) is a platinum compound that was discovered in the 1960s and is one of the most potent chemotherapeutic drugs used for cancer treatment. ${ }^{5,6}$ Its mechanism involves DNA binding and the subsequent activation of multiple signaling pathways to induce cell cycle arrest and apoptosis. These processes have high efficacy for treating a variety of cancer types. ${ }^{7-10}$ However, repeated treatment with cisplatin frequently results in the acquisition of resistance to anti-cancer drugs. ${ }^{10-13}$ This resistance to anti-cancer drugs such as cisplatin is an important contributor of treatment failure in many cancers and represents an unmet clinical need. ${ }^{11,12}$ Thus, many clinical researchers have begun to develop new strategies to overcome this chemo-resistance, including combining platinum-based chemotherapy with molecularly targeted drugs. Indeed, to fulfill this unmet clinical need among cancer patients with cisplatin resistance, studies have tried to provide definitive evidence regarding the mechanisms of the novel drug targets that can be used with cisplatin.

Previously published studies ${ }^{12-18}$ have shown that among the numerous mechanisms of cisplatin resistance, it was necessary to focus on acquired apoptosis inhibition to overcome cisplatin resistance in a wide variety of cancers. In a previous study, we

\footnotetext{
${ }^{1}$ Laboratory of Tumor Immunology, Department of Biomedical Sciences, Graduate School of Medicine, Korea University, Seoul, Korea; ${ }^{2}$ Department of Biochemistry \& Molecular Biology, College of Medicine, Korea University, Seoul, Korea; ${ }^{3}$ Department of Biomedical Science, College of Medicine, Korea University, Seoul, Korea; ${ }^{4}$ Translational Research Institute for Incurable Diseases, College of Medicine, Korea University, Seoul, Korea; ${ }^{5}$ Department of Obstetrics and Gynecology, Gangnam Severance Hospital, Yonsei University College of Medicine, Seoul, Korea and ${ }^{6}$ Experimental Pathology Laboratory, Laboratory of Pathology, Center for Cancer Research, National Cancer Institute, National Institutes of Health, Bethesda, MD, USA

${ }^{7}$ These authors contributed equally to this work.

Correspondence: Professor TW Kim, Laboratory of Tumor Immunology, Korea University College of Medicine, Room 319, Moonsook Medical Hall, 73 Inchon-ro, Sungbuk-gu, Seoul 02841, Republic of Korea.
}

E-mail: twkim0421@Korea.ac.kr

Received 26 December 2016; revised 27 February 2017; accepted 15 March 2017 
showed that resistance to cytotoxic $\mathrm{T}$ lymphocyte-induced apoptosis in cancer cells after immunotherapies was associated with upregulation of the anti-apoptotic gene API5. ${ }^{19-23}$ Furthermore, we discovered that API5 mediates immune resistance by upregulating the FGFR1/ERK pathway, which regulates the levels of the pro-apoptotic molecule Bim. ${ }^{19}$ In addition, another group reported that depleting API5 was shown to enhance the cytotoxic effect of chemotherapeutic drugs. ${ }^{24}$ However, the precise molecular mechanism of API5 activity remains unclear. Nevertheless, we predicted that API5 regulation of the EGFR1/ ERK/Bim axis is closely connected to the acquisition of cisplatin resistance and that it is presumably a critical pathway that could address an unmet clinical need regarding increased incidence of resistance after cisplatin treatment in cancer cells. In patients with cervical cancer, API5 overexpression was closely associated with tumor progression and overall survival. ${ }^{25}$ In addition, there are many reports showing that high levels of API5 were significantly associated not only with tumor stage and grade but also with the chemo-radiotherapy response, and these levels were also associated with p-ERK1/2 in a subset of cervical cancer patients. ${ }^{25,26}$

Here we predicted that API5 levels are critical for the acquisition of cisplatin resistance in human cancer patients. Therefore, in this report, we demonstrated that acquired resistance after repeated treatments with cisplatin is related to high levels of API5 expression and downregulation of the pro-apoptotic molecule Bim via FGFR/ERK signaling in human cancer cells. We aimed to verify via in vivo experiments whether blocking the API5 downstream pathway could be a promising novel strategy for therapeutic intervention.

\section{MATERIALS AND METHODS}

\section{Mice}

Five-week-old female nude mice were purchased from Central Lab. Animal (Seoul, Korea). All mice were maintained and handled according to a protocol approved by the Korea University Institutional Animal Care and Use Committee (KUIACUC-2015-282). All animal procedures were performed in accordance with recommendations for the proper use and care of laboratory animals.

\section{Cells}

CaSki, HeLa and HCT116 cells were commercially obtained from ATCC (Manassas, VA, USA). CaSki and HeLa cells were grown in RPMI 1640. HCT116 cells were cultured in Dulbecco's Modified Eagle Medium. All media were supplemented with $10 \%$ fetal bovine serum, $1 \%$ penicillin and $1 \%$ streptomycin, and all cell lines were cultured at $37^{\circ} \mathrm{C}$ in an atmosphere containing $5 \% \mathrm{CO}_{2}$.

\section{DNA constructs}

The pMSCV-hAPI5 plasmids used in this study have been previously described. ${ }^{19}$

\section{siRNA constructs}

Specific small interfering RNAs (siRNAs) for GFP (\#SP-2003, control), API5 (\#1006580) and FGFR1 (\#100382) were purchased from Bioneer (Daejeon, Korea). The siRNA sequences are as follows: GFP, (sense)
5'-GCA UCA AGG UGA ACU UCA A-3' and (antisense) 5'-GCA UCA AGG UGA ACU UCA A-3'; API5, (sense) 5'GAC CUA GAA CAG ACC UUC A-3' and (antisense) 5'-UGA AGG UCU GUU CUA GGU C-3'; and FGFR1, (sense) 5'CUC ACU GUG GAG UAU CCA U-3' and (antisense) 5'-AUG GAU ACU CCA CAG UGA G-3' The siRNAs were delivered in vitro into six-well plates at a dose of 200 pmol per well using Lipofectamine 2000 (Invitrogen, Gaithersburg, MD, USA).

\section{Cisplatin and SSR128129 treatments}

CaSki, Hela and HCT116 cells were maintained in their respective media containing $10 \%$ fetal bovine serum at $37^{\circ} \mathrm{C}$ with $5 \% \mathrm{CO}_{2}$. For the in vitro treatments, CaSki, Hela and HCT 116 cells were seeded in six-well plates at $1 \times 10^{5}$ cells per well. When the cells grew to $\sim 70 \%$ confluence, the control and cisplatin groups were grown in the presence or absence of $4 \mu \mathrm{M}$ cisplatin for $24 \mathrm{~h}$. For the SSR128129 and cisplatin co-treatment groups, CaSki, Hela and HCT 116 cells were treated with $0.05 \mu \mathrm{M}$ SSR12819 for $8 \mathrm{~h}$ followed by the addition of $2 \mathrm{ml}$ of fresh medium containing $4 \mu \mathrm{g} \mathrm{ml}^{-1}$ cisplatin for $24 \mathrm{~h}$.

\section{Generation of the CaSki P3 CR cancer line}

For cisplatin selection, nude mice were subcutaneously inoculated with $1 \times 10^{6}$ CaSki cells per mouse. At 7 days following the cancer challenge, the mice received cisplatin (Selleckchem, Huston, TX, USA). This treatment regimen was repeated for three cycles.

\section{Flow cytometry analysis}

The P0, P1, P2, P3, N1, N2 and N3 CaSki cancer cell populations were harvested by trypsinization, washed and resuspended in phosphatebuffered saline (PBS). To detect API5 expression, primary antibodies were incubated for $1 \mathrm{~h}$ at $4{ }^{\circ} \mathrm{C}$ and rinsed, after which secondary antibodies were added for $30 \mathrm{~min}$ at $4{ }^{\circ} \mathrm{C}$. The primary antibody used targeted API5 (1:100; Santa Cruz Biotechnology, Santa Cruz, CA, USA), and the secondary antibody was an anti-rabbit $\operatorname{IgG}$ labeled with Alexa 488 (Invitrogen Life Technologies, Carlsbad, CA, USA, \#161314). Data acquisition was performed on a FACSCalibur flow cytometer (BD Biosciences, San Jose, CA, USA) using CellQuest Pro software (BD Biosciences).

\section{Western blot analysis}

Lysates extracted from a total of $1 \times 10^{5}$ cells were used to perform the western blot analysis. Primary antibodies against p-FGFR (Y653/654), FGFR, p-ERK (T202/Y204) and ERK were purchased from Cell Signaling (Danvers, MA, USA) and diluted 1:5000. Antibodies against API5 (1:1000, Santa Cruz Biotechnology) and $\beta$-actin (1: 10 000, MBL, Nagoya, Japan) were used for western blotting, and the appropriate secondary antibodies were conjugated to horseradish peroxidase. Immunoreactive bands were developed using a chemiluminescence ECL detection system (Elpis Biotech, Daejeon, Korea), and the signals were detected using a luminescent image analyzer (LAS-4000 Mini, Fujifilm, Tokyo, Japan). The intensity of the western blot signals was quantified using Multi Gauge software (Fujifilm).

\section{Apoptosis analysis}

To measure drug sensitivity in a cisplatin selection model using human cervical cancer cells, CaSki P0, P1, P2 and P3 as well as N1, N2 and N3 cancer cell populations were harvested by trypsinization and washed once with PBS. After cisplatin selection, the percentage of apoptotic cells 
a

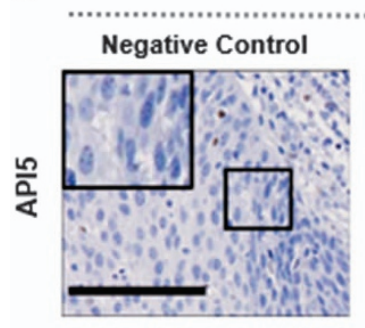

Cervical cancer

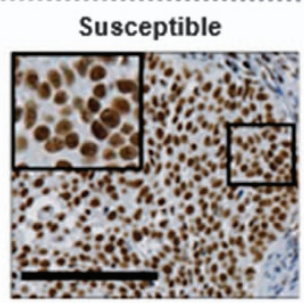

b

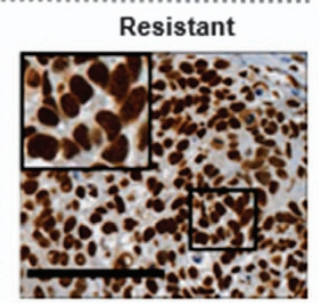

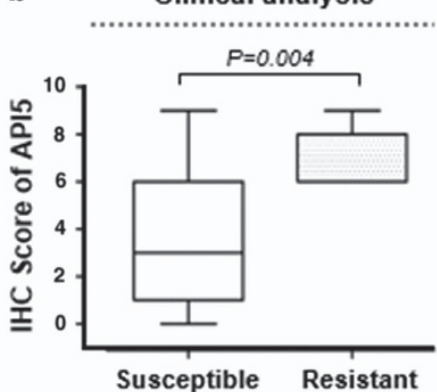

Figure 1 The expression levels of API5 were elevated in human tissues resistant to chemoradiation therapy. (a) Representative immunohistochemical (IHC) staining images of API5 in susceptible and resistant tissues from women with cervical cancer who underwent chemoradiation therapy. Negative controls were processed using a mouse immunoglobulin G (IgG) isotype antibody. Boxed regions indicate high magnification in the insets (scale bar, $100 \mu \mathrm{m}$ ). (b) Box plot depiction of the IHC data. The IHC score is an index of API5 expression and was computed based on the intensity and area of positive staining. Chemoradiation-resistant samples presented significantly higher API5 expression than chemoradiation-susceptible samples (Mann-Whitney U-test, $P=0.004$ ).

was determined by staining with an antibody targeting active caspase-3 and performing flow cytometry as previously described.

\section{Cancer treatment experiments}

On the day of injection, cancer cells were harvested by trypsinization, washed once with Opti-MEM (Gibco BRL, Carlsbad, CA, USA) and resuspended in Opti-MEM to the desired concentration for subcutaneous injection. Nude mice were subcutaneously injected with $5 \times 10^{6}$ CaSki P3 CR cells per mouse. At 12 and 19 days following the challenge, a thermosensitive chitosan hydrogel $(\mathrm{CH})^{27}$ containing either $0.05 \mathrm{mg} \mathrm{kg}^{-1}$ of SSR128129E (Selleckchem) or DMSO was injected into the tumor. Mice were monitored for cancer burden and survival for 35 and 50 days after the initial cancer injections.

\section{Immunohistochemistry}

We previously performed immunohistochemistry for API5 in 152 surgically resected cervical carcinoma samples using tissue microarrays and compared the data with clinicopathological factors, including the survival rates of patients with cervical cancer. ${ }^{25}$ The samples were collected from patients with cervical cancer who were treated at the Gangnam Severance Hospital between 1996 and 2010. Some of the paraffin blocks were provided by the Korea Gynecologic Cancer Bank through the Bio \& Medical Technology Development Program of the Ministry of Education, Science and Technology, Korea (NRF-2012M3A9B8021800). The tissue specimens and medical records were obtained with informed consent from all included patients and approval of the local research ethics committee (Seoul, South Korea). To assess API5 staining, two scores were assigned to each sample: (a) staining intensity (categorized as 0 (absent), 1 (weak), 2 (moderate) or 3 (strong)) and (b) the percentage of positively stained epithelial cells (scored as 0 (0\% positive), 1 (1-25\%), 2 (26-50\%), $3(51-75 \%)$ and $4(76-100 \%))$. A histoscore was generated by multiplying the mean intensity and percent scores (overall score range $0-12$ ). After reexamining the statistical significance between groups susceptible and resistant to chemoradiation based on previous immunohistochemical data of API5, we generated new figures and graphs.

\section{Statistical analysis}

All data are representative of at least three separate experiments. Individual data points were compared using a two-tailed Student's $t$ test. For the IHC data, statistical analysis was performed using SPSS version 21 (SPSS, Chicago, IL, USA). The Mann-Whitney $U$-test was used to compare the protein expression levels between each group.

\section{RESULTS}

High levels of API5 correlate with resistance to chemoradiation therapy in patients with cervical cancer

We previously demonstrated via immunohistochemistry that API5 protein expression was significantly associated with cervical cancer progression. ${ }^{25}$ As observed in the previous report, API5 was exclusively localized to the nucleus of tumor cells (Figure 1a). We found that API5 expression was positively correlated with resistance to chemoradiation therapy $(P=0.004)$ (Figure $1 \mathrm{~b})$. These results indicated that the API5 expression levels were highly correlated with chemo-resistance in human cervical cancer.

Tumor cells with high levels of API5 are selected by repeated treatment with cisplatin

Given that API5 has been defined as a negative prognostic factor in cervical cancer and has been implicated as an inhibitor of chemoradiation therapy in cervical cancer, we decided to further examine the role of API5 regarding chemoradiation resistance. First, to examine the biological role of API5 in the chemo-resistance of cervical cancer cells, we applied a cisplatin selection model using a human cervical cancer cell line as illustrated in Figure 2a. Human cervical cancer cells from the CaSki line (designated as P0 cells) were treated with cisplatin. Live cancer cells were recovered as the P1 line. Further rounds of selection were used to generate the $\mathrm{P} 2$ and $\mathrm{P} 3$ populations. In addition, we confirmed that the populations exhibited sequentially increasing resistance to cisplatin treatment as demonstrated by the percentage of active caspase $-3^{+}$cells. Strikingly, $\sim 20 \%$ of the P3 cells survived upon treatment with cisplatin compared with $\sim 3 \%$ of the P0 cells (Figure $2 \mathrm{~b}$ ). On the other hand, the number of surviving cancer cells from N1 to N3, which were parallel stages without cisplatin selection, remained constant, and their percentage of surviving cells were similar to that of P0 cells treated in the 
a

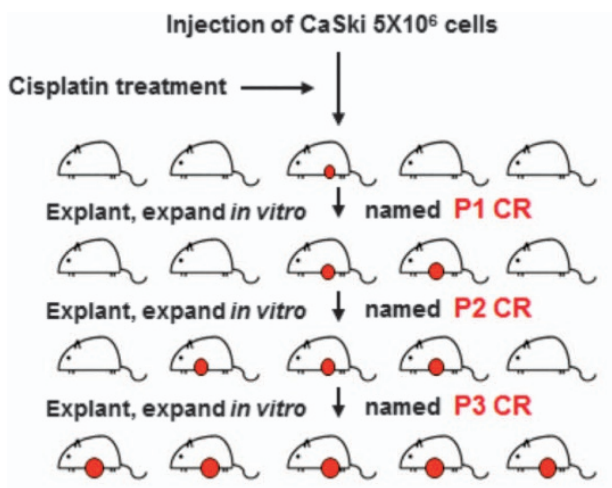

b

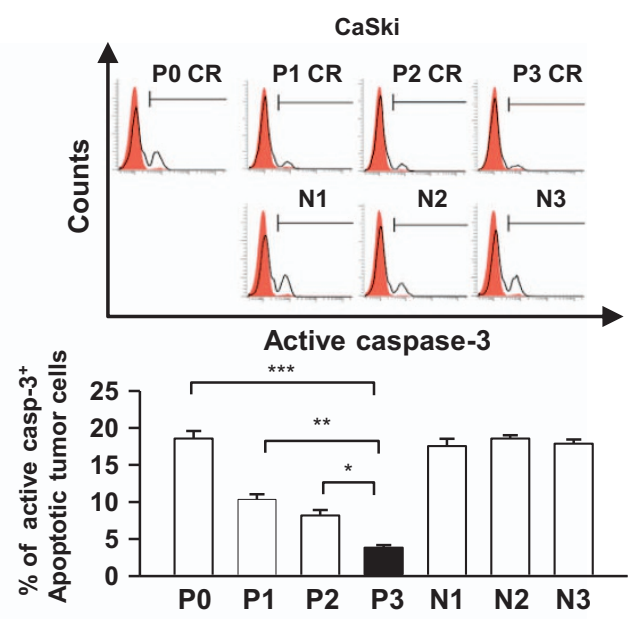

d

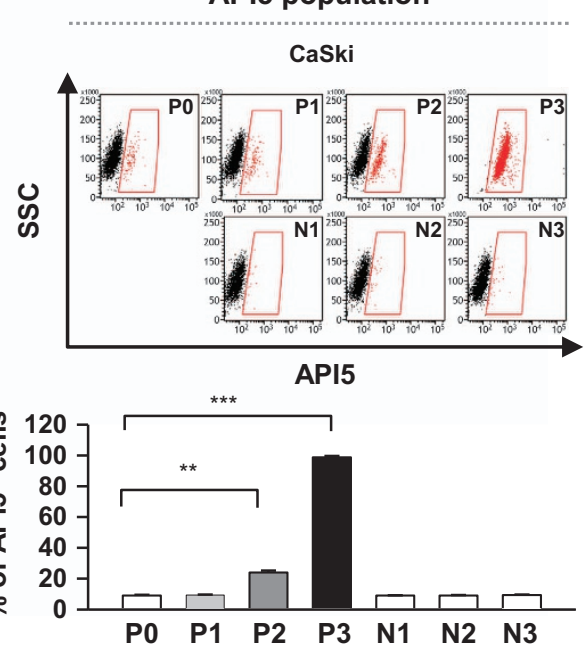

Figure 2 Cisplatin selection enriches the API5 high cell population of human cancer cells. (a) The diagram depicting the process of in vivo cisplatin selection among human cervical cancer cells. (b-d) Each experiment was performed in cancer cells at each stage with cisplatin-selected (P0, P1, P2 and P3 populations) and naïve cells (N1, N2 and N3 populations). (b) Flow cytometry analysis of the percentage of apoptotic (active caspase- $3^{+}$) cells after treatment with $4 \mu \mathrm{m}$ cisplatin for $36 \mathrm{~h}$. (c) Western blot analysis of API5 expression. $\beta$-actin was included as an internal loading control. The numbers below the blot images indicate the expression as measured by fold change. Top, Quantification of API5 expression. Bottom, representative western blot images. (d) Flow cytometry analysis of cells expressing high levels of API5. Top, Representative FACS images. Bottom, Quantification of the frequency of cells with high levels of API5 expression. All the graphs represent three independent experiments performed in triplicate. Error bars represent the s.d.'s from the mean. ${ }^{*} P<0.01,{ }^{* *} P<0.005$, $* * * P<0.001$.

same manner (Figure 2b). Using these models, we assessed API5 expression to determine whether the API5 expression levels were correlated with cisplatin resistance. As a result, we observed a consistent and gradual increase in API5 expression from $\mathrm{P} 0$ to $\mathrm{P} 3$, but there were no changes in the API5 expression levels in the cancer cell populations from N1 to N3 (Figure 2c). Remarkably, the total level of API5 protein was eight-fold higher in P3 cells than in P0 cells (Figure 2c). The overall increase in API5 expression in the P3 line was likely due to the enrichment of API $5^{\text {high }}$ cells as opposed to upregulation of API5 because the percentage of API5 $5^{\text {high }}$ cells rose from $\sim 5 \%$ in the P0 line to $\sim 90 \%$ in the P3 CR line (Figure $2 \mathrm{~d}$ ).
Thus, these data indicated that repeated cisplatin therapy may induce drug resistance by enriching the API $5^{\text {high }}$ cell population.

API5 induces downregulation of the apoptotic molecule Bim via FGFR1-ERK signaling to cause cisplatin resistance in multiple types of API5-overexpressing human cancers To examine the molecular basis of enhanced cisplatin resistance in cancer cells following cisplatin selection, we assessed the expression of a set of pro- and anti-apoptotic molecules from the P0 and P3 CR populations. The protein levels of Xiap, $\mathrm{Bcl} 2$ and $\mathrm{Bcl}_{\mathrm{xl}}$ (as anti-apoptotic molecules) and of Bad, Bak, 
a

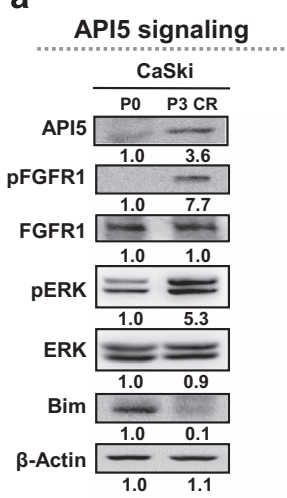

b

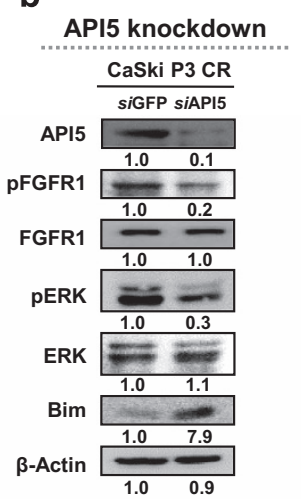

c

Cisplatin induced apoptosis

CaSki P3 CR

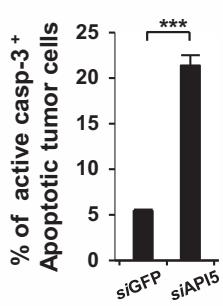

d

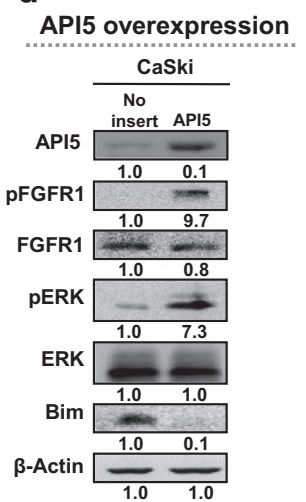

e

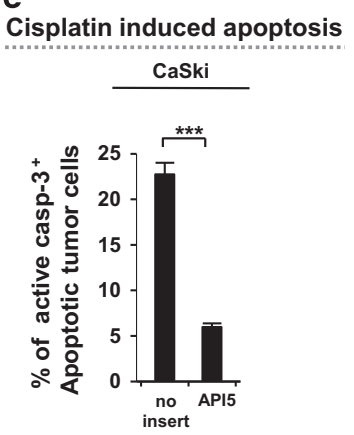

f

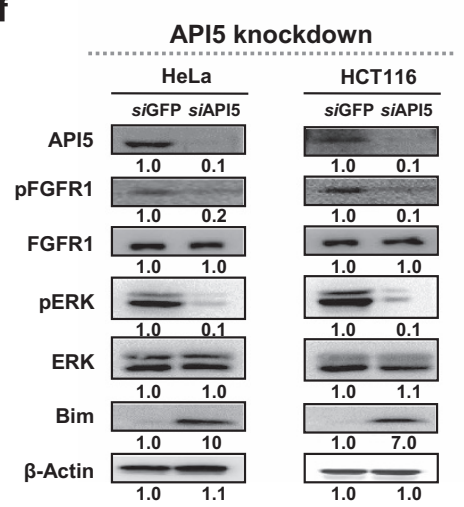

g

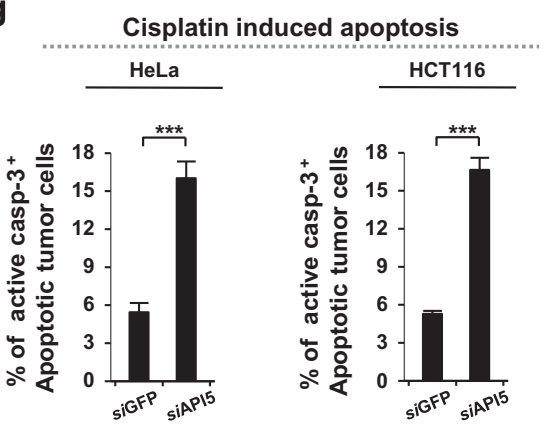

Figure 3 API5 promotes cisplatin resistance in human cancer cells through the FGFR1-ERK-Bim pathway. (a, b, d and f) Western blot analysis of the expression and activation status (a) of pFGFR, pERK and Bim in PO or P3 CR cells, (b) in P3 CR cells treated with either siGFP or siAPI5, (d) in empty vector- or API5 cDNA-transfected PO cells and (f) in HeLa and HCT116 cells treated with either siGFP or siAPI5. $\beta$-actin was included as an internal loading control. The numbers below the blot images indicate the expression as measured by fold change. (c, e and $\mathbf{g}$ ) Flow cytometry analysis of the percentage of apoptotic (active caspase- $3^{+}$) cells (c) in P3 CR cells treated with either siGFP or siAPI5, (e) in empty vector- or API5 cDNA-transfected PO cells and (g) in HeLa and HCT116 cells treated with either siGFP or siAPI5. All the graphs represent three independent experiments performed in triplicate. Error bars represent the s.d.'s from the mean. ${ }^{* * *} P<0.001$.

Bax and Bid (as pro-apoptotic molecules) were comparable between the CaSki P0 and CaSki P3 CR cell lines (Supplementary Figure 1a). However, the expression of Bim, a pro-apoptotic protein, was substantially diminished in CaSki P3 CR cells (Supplementary Figure 1a). A similar result was observed in our previous report, which stated that API5 rendered cancer cells resistant to cytotoxic $\mathrm{T}$ lymphocyteinduced apoptosis via downregulation of Bim by activating the FGFR1 signaling pathway. ${ }^{19}$ Therefore, we observed the involvement of FGFR1 signaling in the acquisition of cisplatin resistance. To assess whether the established cisplatin-resistant cells trigger the FGFR1 pathway, we evaluated the activation of FGFR1 and ERK by measuring their respective phosphorylation levels in CaSki P0 and P3 CR cells. As expected, FGFR1 and ERK phosphorylation levels were markedly increased in CaSki P3 CR cells (Figure 3a).

We next investigated whether upregulated API5 levels in CaSki P3 CR cells activated FGFR1/ERK signaling to promote cisplatin resistance. We treated CaSki P3 CR cells with siRNA targeting either API5 (siAPI5) or GFP (siGFP) control and observed the downstream signaling of FGFR1 as well as the sensitivity toward cisplatin resistance. The knockdown efficiency was confirmed by western blot analysis (Figure 3b). Inhibiting API5 expression drastically decreased the phosphorylation of FGFR and ERK by $\sim 3$ - to 5 -fold and increased the protein levels of Bim by approximately eight-fold in the CaSki P3 CR cells (Figure 3b). Consistent with these changes in the protein levels, upon cisplatin administration, siAPI5-treated cells had nearly four-fold more apoptotic cells than the siGFPtreated cells (Figure 3c). Conversely, we confirmed that introduction of API5 into CaSki P0 CR cells increased the phosphorylation levels of FGFR1 and ERK by more than approximately 7 -fold and decreased the Bim protein levels by 10-fold (Figure 3d); furthermore, cisplatin treatment resulted in nearly 4-fold fewer apoptotic API5-overexpressing cells than non-transduced cells (Figure 3e). We further confirmed whether the API5-FGFR1-Bim axis is conserved across multiple cancers. In a previous report, we profiled API5 expression across a variety of human cancer cell lines and found high levels of API5 in several breast, lung, ovarian and colon cancer cell lines such as HeLa and HCT116. ${ }^{19}$ Therefore, we selected HeLa and HCT116 cells as endogenous API-5 high 
cancer cell lines and transfected them with either siAPI5 or siGFP to examine the downstream FGFR1 signaling and the changes in chemo-resistance to cisplatin. The knockdown efficiency of API5 in the two cancer cell lines was confirmed by western blot analysis (Figure 3f). Inhibition of API5 expression drastically decreased the levels of phosphorylated FGFR and ERK by approximately 5- and 10-fold, respectively, and increased the Bim protein levels in both HeLa and HCT116 cells by approximately 10- and 7-fold, respectively (Figure 3f). Consistent with this, siAPI5-transfected cells treated with cisplatin had approximately three times more apoptotic cells than treated siGFP-transfected cells (Figure $3 \mathrm{~g}$ ). Therefore, these data indicated that API5 conveys resistance to cancer cells and contributes to their cisplatin-resistant phenotype via the FGFR1/ERK/Bim axis.

\section{API5-mediated resistance to cisplatin can overcome inhibition of the FGFR1 pathway}

We would expect that blocking the API5 pathway could be a promising approach for treating cisplatin-mediated resistant human cancers. However, there are no pharmacological inhibitors of API5 available. Therefore, we took note of FGFR1, a downstream effector of the API5 pathway. As FGFR1 signaling plays an important role in the API5-mediated resistant phenotypes of cancer cells, we would expect that inhibiting FGFR1 signaling may be an effective strategy in cancer cells with API5-mediated cisplatin resistance. To test this hypothesis, we examined whether API5-mediated cisplatin resistance can be overcome by treatment with SSR128129E, an allosteric inhibitor of FGF receptor signaling at nanomolar concentrations. In API5-overexpressing cells, SSR128129E treatment drastically decreased the levels of phosphorylated FGFR1 and ERK by 8 - to 10-fold and increased the Bim protein levels by more than 2-fold (Figure 4a). Consistent with these changes in the protein levels, in the presence of cisplatin, there were nearly 5-fold more apoptotic SSR128129E-treated cells than DMSO-treated cells (Figure 4b).

Then, we confirmed whether these features were mediated by FGFR1 specificity during the change in susceptibility to API5-mediated cisplatin resistance via inhibition of the FGF receptor by SSR128129E. We treated CaSki P3 CR cells with siRNA targeting either FGFR (siFGFR) or GFP (siGFP, control). We observed that administration of siFGFR decreased the pERK protein levels by approximately 3-fold and increased the Bim levels by more than 5-fold in the CaSki P3 CR line (Figure 4c). Consistent with these changes in the protein levels, in the presence of cisplatin, there were nearly 5-fold more apoptotic siFGFR-treated cells than siGFP-treated cells (Figure 4d). Thus, our data showed that inhibition of FGFR1 by SSR128129E represents an attractive strategy to overcome API5-mediated cisplatin resistance in human cervical cancer.

To overcome cisplatin resistance, inhibiting the FGFR1 pathway is an efficient approach in multiple types of API5overexpressing human cancers with cisplatin resistance To verify the phenotypic effects of SSR128129E in different types of API5-overexpressing cancers, we selected three representative lines, HeLa and HCT116 cells (from patients with cervical and colon cancer, respectively) as well as CaSki P3 CR cells (which overexpressed API5 and exhibited a cisplatin-resistant phenotype) and treated cells with either cisplatin or SSR128129E. As expected, treatment with SSR128129E inhibited FGFR1 activity in all the tested cancer cell lines in which FGFR1 signaling was enhanced by API5, which led to inhibition of ERK activity and an increase in the Bim protein levels (Figure 5a). In addition, consistent with these changes in the protein levels, there were 4to 9-fold more apoptotic SSR128129E-treated cells than DMSOtreated cells (Figure 5b). Interestingly, we found a striking increase in the Bim protein levels after a combination treatment with cisplatin and SSR128129E than after treatment with a

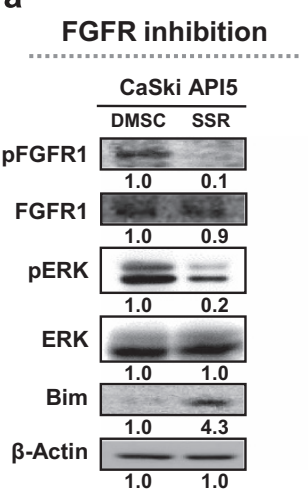

b

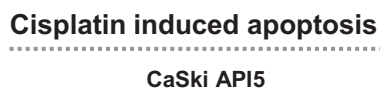

C

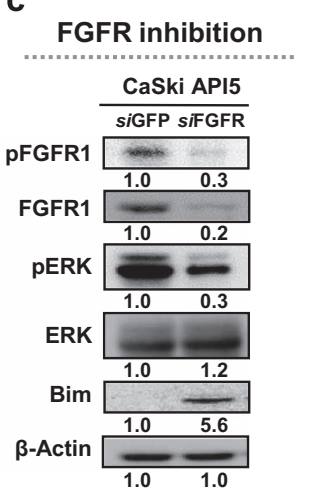

d Cisplatin induced apoptosis CaSki API5

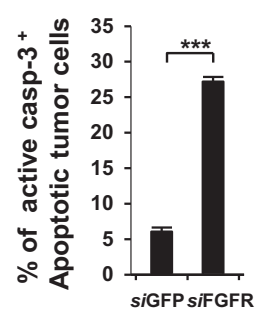

Figure 4 Inhibition of FGFR1 disrupts resistance in API5-mediated cisplatin-resistant cells. (a, c) Western blot analysis of the expression and activation status (a) of pFGFR, pERK and Bim after treatment of API5-overexpressing cells (a) with either DMSO or SSR128129E and (c) with either siGFP or siAPI5. $\beta$-actin was included as an internal loading control. The numbers below the blot images indicate the expression as measured by fold change. (b, d) Flow cytometry analysis of the percentage of apoptotic (active caspase- $3^{+}$) cells after treatment of API5-overexpressing cells (b) with either DMSO or SSR128129E or (d) with either siGFP or siAPI5. All the graphs represent three independent experiments performed in triplicate. Error bars represent the s.d.'s from the mean. ${ }^{* * *} P<0.001$. 
a

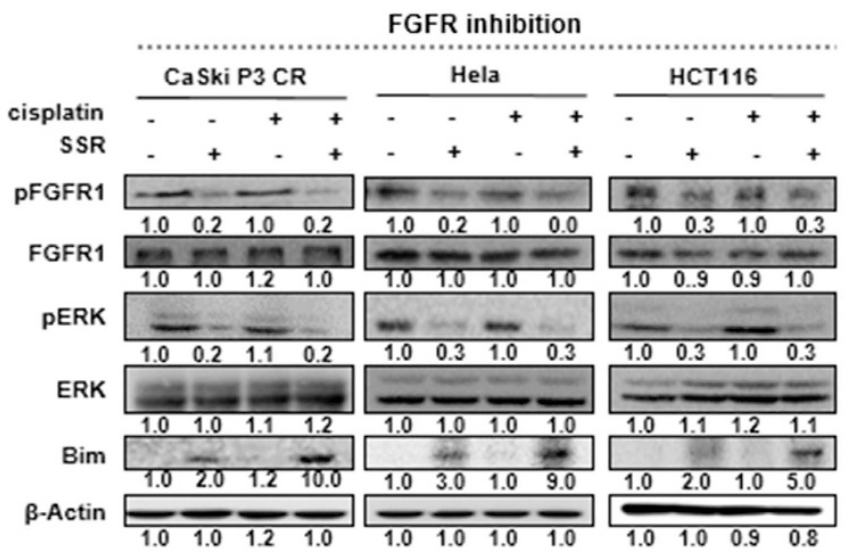

b

Cisplatin induced apoptosis
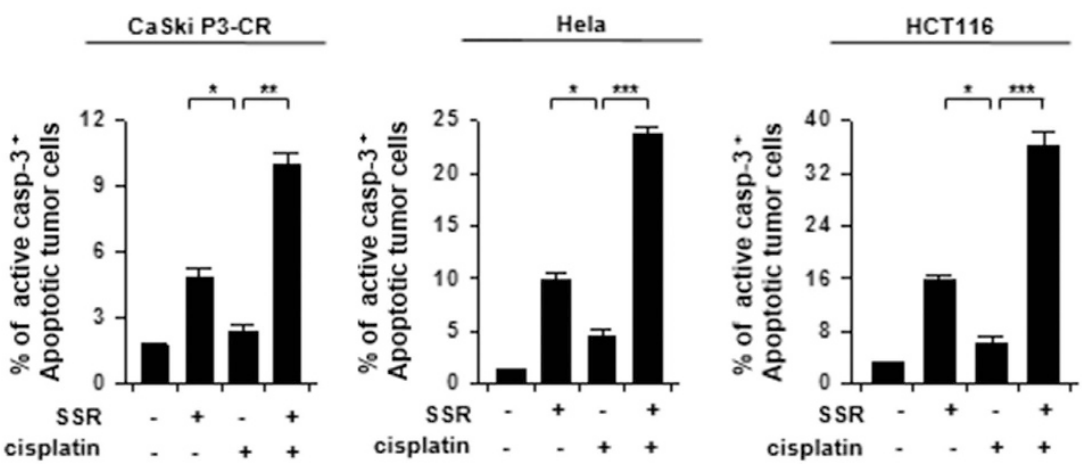

Figure 5 FGFR1 signaling mediates cisplatin resistance in multiple types of human cancer cells that overexpress API5. (a) Western blot analysis of the expression and activation status of pFGFR, pERK and Bim after treatment of CaSki P3 CR, HeLa and HCT116 cells with DMSO, SSR128129E or cisplatin. $\beta$-actin was included as an internal loading control. The numbers below the blot images indicate the expression as measured by fold change. (b) Flow cytometry analysis of the percentage of apoptotic (active caspase- $3^{+}$) cells after treatment of CaSki P3 CR, HeLa and HCT116 cells with DMSO, SSR128129E or cisplatin. All the graphs represent three independent experiments performed in triplicate. Error bars represent the s.d.'s from the mean. ${ }^{*} P<0.01,{ }^{* *} P<0.001,{ }^{* * *} P<0.001$.

SSR128129E alone (Figure 5a, line 5). These results parallel the marked increase in the percentage of apoptotic cells under the same conditions (Figure 5b, column 4, respectively). Here, we confirmed that treatment with SSR128129E to target FGFR1 signaling could be a common strategy in human cancers with API5-mediated cisplatin resistance; moreover, this drug can sensitize cells and improve the therapeutic effects of conventional cisplatin treatment for cancer.

In a preclinical tumor model, SSR128129E leads to the regression of cisplatin-resistant cancer growth

To demonstrate the therapeutic value of the API5 pathway and its downstream molecular axis in vivo, the efficacy of SSR128129E was tested in nude mice bearing CaSki P3 CR cells. As indicated in the schedule described in Figure 6a, mice received cisplatin along with SSR128129E. Treatment with SSR128129E elicited a profound therapeutic effect, and when it was used in combination with cisplatin, the cancer was virtually undetectable even at 21 days after tumor challenge (Figure $6 \mathrm{~b}$ and $\mathrm{d}$ ). Importantly, 100\% of mice that received both cisplatin and SSR128129E survived, even at 48 days after tumor challenge, while all the animals in the other groups had died (Figure 6c). Consistent with the in vitro results, we observed reduced levels of phosphorylated FGFR1 and ERK as well as elevated levels of Bim protein in the tumor tissues from all the SSR128129E-treated mice compared with tumor tissues from the PBS-treated mice (Figure 6e). In addition, the overall cytotoxic effect of cisplatin was greater after SSR128129E treatment relative to that after treatment with PBS as indicated by the percentage of apoptotic tumor cells (Figure 6f). Taken together, these data showed that inhibiting FGFR1 represents a rational applicable strategy for regulating API5 $5^{\text {high }}$ cisplatin-resistant human cancers.

\section{DISCUSSION}

Resistance to anti-cancer drugs is a critical problem in chemotherapy for many cancers and is a major cause of treatment failure in patients undergoing chemotherapy. Clinically, a treatment strategy that includes cisplatin among the anti-cancer drugs is implemented in a variety of cancer types. $^{2-4,6}$ Cisplatin has a high risk of drug resistance development although it has been shown to have a high 
a
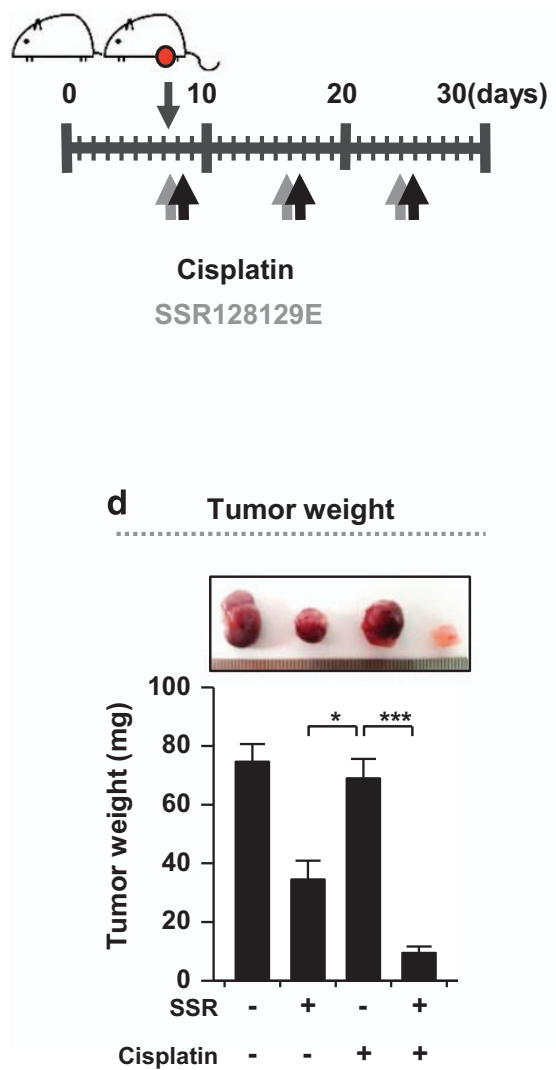

b

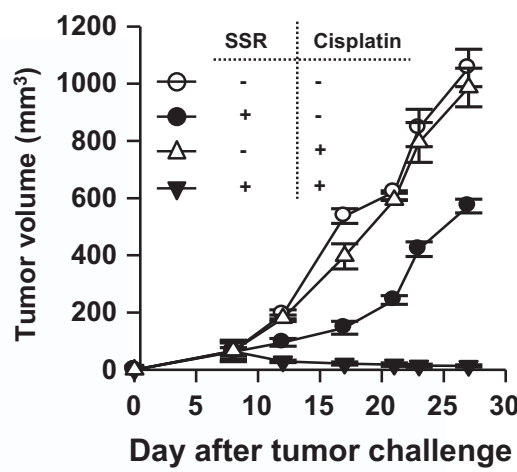

e

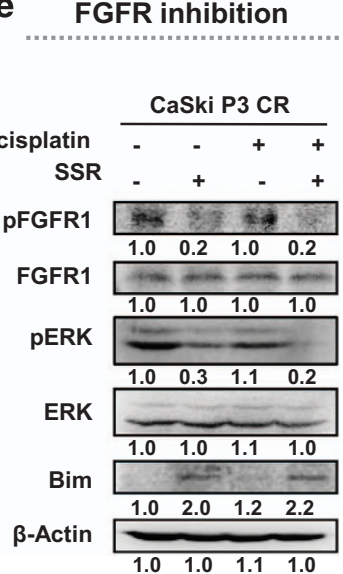

c Survival

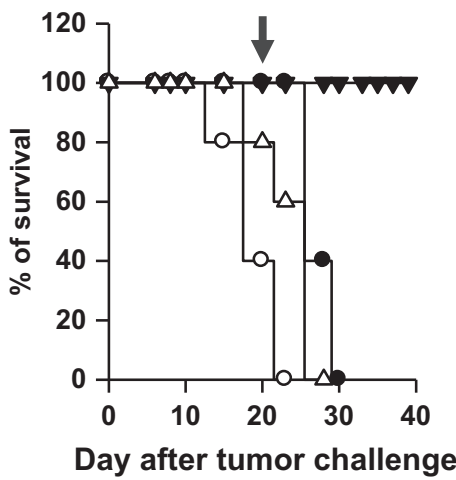

f Cisplatin induced apoptosis

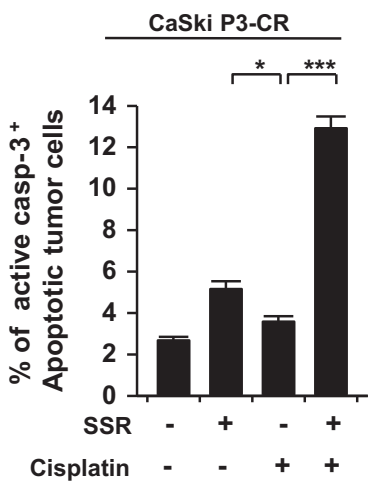

Figure 6 Inhibition of FGFR1 renders the cancer cells susceptible to cisplatin-mediated cytotoxicity. (a) Schematic of the therapy regimen in mice implanted with CaSki P3 CR cervical cancer cells. (b) Cancer growth and (c) survival of mice inoculated with CaSki P3 CR cells treated with the indicated regimens (8 mice per group). (d) Cancer mass of mice at 21 days after inoculation. (e) Western blot analysis of pFGFR, pERK and Bim expression in mice administered either PBS or SSR128129E in the presence or absence of cisplatin. $\beta$-actin was included as an internal loading control. The numbers below the blot images indicate the expression as measured by fold change. (f) Flow cytometry analysis of the percentage of apoptotic (active caspase- $3^{+}$) cells in the cancer tissues of mice treated with either PBS or SSR128129E in the presence or absence of cisplatin. Error bars represent the s.d.'s from the mean. ${ }^{*} P<0.01,{ }^{*} P<0.005$, $* * * P<0.001$.

chemotherapeutic efficacy. ${ }^{2}$ If researchers are unable to define the mechanism of acquired resistance to treatment with cisplatin, many cancer patients who receive cisplatin treatment will be at various risks of developing drug resistance. In this respect, this study showed that the expression pattern in human cancer cells changed after repeated cisplatin treatments. Specifically, in a paper reporting that API5 expression is correlated with a poor prognosis in patients with cervical cancer, it was briefly explained that API5 expression is positively related to resistance to chemo-radiation therapy in patients with cervical cancer. ${ }^{25}$ These findings suggest the possibility that the effect of chemo-radiation therapy can be compromised in cervical cancer patients with API5 overexpression, an observation that can have profound clinical implications.

Here our study provided further evidence to satisfy an unmet clinical need. We elucidated the specific molecular mechanism for the close correlation between API5 overexpression and acquired resistance to cisplatin treatment. At first, the tumor cell population with high API5 expression levels steadily increased during the process of establishing a therapeutic resistance model using cisplatin (Figure 1). This result indicated that API5-overexpressing tumor cells are selected by repeated cisplatin treatments and develop resistance to this drug. There is evidence regarding the intimate relationship between API5 and cisplatin resistance. In addition, the crucial API5/FGFR/ERK axis that confers cisplatin resistance provides important clues suggesting that targeting molecules within the API5 downstream pathway can help overcome cisplatin resistance.

API5 contains protein-protein interaction domains such as HEAT and ARM repeats that are well suited for interactions with multiple binding partners. ${ }^{28}$ Thus, discovering an API5 inhibitor using structure-based drug design studies is possible. However, there are no small-molecule drugs that directly target API5 to date. Considering this, we took note of an inhibitor of 
API5-mediated FGFR activity, which is a receptor tyrosine kinase that is part of a major class of drug targets, as a potential inhibitor of API5 signaling. ${ }^{29-32}$ In a recent report, the smallmolecule allosteric inhibitor SSR128129E, which blocks FGFR signaling at nanomolar doses, was identified, ${ }^{33}$ and its therapeutic potential has been verified in immuno-resistant cancer cells. ${ }^{34}$ It was determined whether this drug can attenuate the API5-mediated resistant properties induced by cisplatin in various types of aggressive cancer cells with high levels of API5 expression. The results were as predicted-when SSR128129E was used in combination with cisplatin in cisplatin-resistant API5 $5^{\text {high }}$ cells, we observed that the cisplatin-mediated resistant phenotype disappeared. The observed synergistic effect of the combination treatment with cisplatin and SSR128129E is shown in Figure 5. This phenomenon may ultimately be driven by a dramatic increase in caspase 3 and Bim levels. Several studies have shown that cisplatin sensitivity is mediated by either Bim or caspase 3 regulation. ${ }^{35,36}$ Thus, our results can be interpreted as a convergence of necrotic features via accumulation of cisplatin-induced apoptotic signals in cells with increased sensitivity to cisplatin via inhibition of FGFR signaling by SSR128129E.

Clinically, a therapeutic strategy with cisplatin that is effective against multiple types of cancers is necessary to minimize cisplatin resistance. In our study, we also conducted in vivo experiments to verify the potential of clinical application of this novel therapeutic strategy. We confirmed that SSR128129E significantly increased the therapeutic efficacy in an established xenograft mouse model using cisplatin-resistant human cancer cells when SSR128129E was used in combination with cisplatin. Thus, we consider it appropriate that during cisplatin-based chemotherapy, FGFR-targeted strategies are useful to treat refractory cancers as well as cisplatin-resistant cancers with high levels of API5 expression.

\section{CONFLICT OF INTEREST}

The authors declare no conflict of interest.

\section{ACKNOWLEDGEMENTS}

This work was funded by the National Research Foundation of Korea (NRF-2014R1A2A1A10054205, NRF-2013M3A9D3045881 and NRF-2013R1A1A2063031).

1 DeVita VT Jr, Chu E. A history of cancer chemotherapy. Cancer Res 2008; 68: 8643-8653.

2 Florea AM, Busselberg D. Cisplatin as an anti-tumor drug: cellular mechanisms of activity, drug resistance and induced side effects. Cancers 2011; 3: 1351-1371.

3 Shah N, Dizon DS. New-generation platinum agents for solid tumors. Future Oncol 2009; 5: 33-42.

4 Zhang J, Wang L, Xing Z, Liu D, Sun J, Li X et al. Status of bi- and multinuclear platinum anticancer drug development. Anticancer Agents Med Chem 2010; 10: 272-282.

5 Rosenberg B, Vancamp L, Krigas T. Inhibition of cell division in Escherichia coli by electrolysis products from a platinum electrode. Nature 1965; 205 : 698-699.
6 Chen D, Milacic V, Frezza M, Dou QP. Metal complexes, their cellular targets and potential for cancer therapy. Curr Pharm Des 2009; 15 : 777-791.

7 Sherman SE, Gibson D, Wang AH, Lippard SJ. X-ray structure of the major adduct of the anticancer drug cisplatin with DNA: cis-[Pt(NH3)2(d(pGpG))]. Science 1985; 230: 412-417.

8 Eastman A. Activation of programmed cell death by anticancer agents: cisplatin as a model system. Cancer Cells 1990; 2: 275-280.

9 Jordan P, Carmo-Fonseca M. Molecular mechanisms involved in cisplatin cytotoxicity. Cell Mol Life Sci 2000; 57: 1229-1235.

10 Siddik ZH. Cisplatin: mode of cytotoxic action and molecular basis of resistance. Oncogene 2003; 22: 7265-7279.

11 Brabec V, Kasparkova J. Modifications of DNA by platinum complexes. Relation to resistance of tumors to platinum antitumor drugs. Drug Resist Updat 2005; 8: 131-146.

12 Sedletska Y, Giraud-Panis MJ, Malinge JM. Cisplatin is a DNA-damaging antitumour compound triggering multifactorial biochemical responses in cancer cells: importance of apoptotic pathways. Curr Med Chem Anticancer Agents 2005; 5: 251-265.

13 Kartalou M, Essigmann JM. Mechanisms of resistance to cisplatin. Mutat Res 2001; 478: 23-43.

14 Ralph SJ, Neuzil J. Mitochondria as targets for cancer therapy. Mol Nutr Food Res 2009; 53: 9-28.

15 Park CM, Park MJ, Kwak HJ, Moon SI, Yoo DH, Lee HC et al. Induction of p53-mediated apoptosis and recovery of chemosensitivity through p53 transduction in human glioblastoma cells by cisplatin. Int J Oncol 2006; 28: 119-125.

16 Xie SY, Li YJ, Wang PY, Jiao F, Zhang S, Zhang WJ. miRNA-regulated expression of oncogenes and tumor suppressor genes in the cisplatininhibited growth of K562 cells. Oncol Rep 2010; 23: 1693-1700.

17 Shen DW, Pouliot LM, Hall MD, Gottesman MM. Cisplatin resistance: a cellular self-defense mechanism resulting from multiple epigenetic and genetic changes. Pharmacol Rev 2012; 64: 706-721.

18 Dai Y, Jin S, Li X, Wang D. The involvement of Bcl-2 family proteins in AKTregulated cell survival in cisplatin resistant epithelial ovarian cancer. Oncotarget 2017; 8: 1354-1368.

19 Noh KH, Kim SH, Kim JH, Song KH, Lee YH, Kang TH et al. API5 confers tumoral immune escape through FGF2-dependent cell survival pathway. Cancer Res 2014; 74: 3556-3566.

20 Noh KH, Kang TH, Kim JH, Pai SI, Lin KY, Hung CF et al. Activation of Akt as a mechanism for tumor immune evasion. Mol Ther 2009; 17: 439-447.

21 Noh KH, Kim BW, Song KH, Cho H, Lee YH, Kim JH et al. Nanog signaling in cancer promotes stem-like phenotype and immune evasion. J Clin Invest 2012; 122: 4077-4093.

22 Noh KH, Lee YH, Jeon JH, Kang TH, Mao CP, Wu TC et al. Cancer vaccination drives Nanog-dependent evolution of tumor cells toward an immune-resistant and stem-like phenotype. Cancer Res 2012; 72: 1717-1727.

23 Mao CP, Wu T, Song KH, Kim TW. Immune-mediated tumor evolution: Nanog links the emergence of a stem like cancer cell state and immune evasion. Oncoimmunology 2014; 3: e947871.

24 Rigou P, Piddubnyak V, Faye A, Rain JC, Michel L, Calvo F et al. The antiapoptotic protein AAC-11 interacts with and regulates Acinus-mediated DNA fragmentation. EMBO J 2009; 28: 1576-1588.

25 Cho H, Chung JY, Song KH, Noh KH, Kim BW, Chung EJ et al. Apoptosis inhibitor-5 overexpression is associated with tumor progression and poor prognosis in patients with cervical cancer. BMC Cancer 2014; 14: 545.

26 Song KH, Kim SH, Noh KH, Bae HC, Kim JH, Lee HJ et al. Apoptosis inhibitor 5 increases metastasis via Erk-mediated MMP expression. BMB Rep 2015; 48: 330-335.

27 Han HD, Song CK, Park YS, Noh KH, Kim JH, Hwang T et al. A chitosan hydrogel-based cancer drug delivery system exhibits synergistic antitumor effects by combining with a vaccinia viral vaccine. Int J Pharm 2008; 350: 27-34.

28 Han BG, Kim KH, Lee SJ, Jeong KC, Cho JW, Noh KH et al. Helical repeat structure of apoptosis inhibitor 5 reveals protein-protein interaction modules. J Biol Chem 2012; 287: 10727-10737.

29 Overington JP, Al-Lazikani B, Hopkins AL. How many drug targets are there? Nat Rev Drug Discov 2006; 5: 993-996.

30 Bono F, De Smet F, Herbert C, De Bock K, Georgiadou M, Fons P et al. Inhibition of tumor angiogenesis and growth by a small-molecule multi-FGF receptor blocker with allosteric properties. Cancer Cell 2013; 23: 477-488.

31 Gasparini G, Longo R, Toi M, Ferrara N. Angiogenic inhibitors: a new therapeutic strategy in oncology. Nat Clin Pract Oncol 2005; 2: 562-577. 
32 Zhang J, Yang PL, Gray NS. Targeting cancer with small molecule kinase inhibitors. Nat Rev Cancer 2009; 9: 28-39.

33 Herbert C, Schieborr U, Saxena K, Juraszek J, De Smet F, Alcouffe C et al. Molecular mechanism of SSR128129E, an extracellularly acting, smallmolecule, allosteric inhibitor of FGF receptor signaling. Cancer Cell 2013; 23: 489-501.

34 Song KH, Cho H, Kim S, Lee HJ, Oh SJ, Woo SR et al. API5 confers cancer stem cell-like properties through the FGF2-NANOG axis. Oncogenesis 2017; 6: e285.

35 Wang J, Zhou JY, Wu GS. Bim protein degradation contributes to cisplatin resistance. J Biol Chem 2011; 286: 22384-22392.

36 Henkels KM, Turchi JJ. Cisplatin-induced apoptosis proceeds by caspase3-dependent and -independent pathways in cisplatin-resistant and -sensitive human ovarian cancer cell lines. Cancer Res 1999; 59: 3077-3083.

Supplementary Information accompanies the paper on Experimental \& Molecular Medicine website (http://www.nature.com/emm) (c) (i) (s) $\odot$ This work is licensed under a Creative Commons Attribution-NonCommercial-NoDerivs $\quad 4.0$

International License. The images or other third party material in this article are included in the article's Creative Commons license, unless indicated otherwise in the credit line; if the material is not included under the Creative Commons license, users will need to obtain permission from the license holder to reproduce the material. To view a copy of this license, visit http://creativecommons.org/licenses/by-nc-nd/4.0/

(C) The Author(s) 2017 